\title{
Tandem processes promoted by a hydrogen shift in 6-aryl- fulvenes bearing acetalic units at ortho position: a combined experimental and computational study
}

\author{
Mateo Alajarin ${ }^{1}$, Marta Marin-Luna ${ }^{1}$, Pilar Sanchez-Andrada ${ }^{*}$ and Angel Vidal ${ }^{1}$
}

\author{
Full Research Paper \\ Address: \\ ${ }^{1}$ Departamento de Química Orgánica, Universidad de Murcia, \\ Facultad de Química, Regional Campus of International Excellence \\ "Campus Mare Nostrum", Espinardo, 30100 Murcia (Spain) and \\ 2University Centre of Defence at the Spanish Air Force Academy, \\ Base Aerea de San Javier, C/ Coronel López Peña s/n, 30720, \\ Santiago de la Ribera, Murcia, Spain \\ Email: \\ Pilar Sanchez-Andrada* - pilar.sanchez@cud.upct.es \\ * Corresponding author

\section{Keywords:} \\ acetal; benzindenes; DFT calculations; fulvene; hydrogen shift
}

Beilstein J. Org. Chem. 2016, 12, 260-270.

doi:10.3762/bjoc. 12.28

Received: 14 December 2015

Accepted: 31 January 2016

Published: 11 February 2016

Associate Editor: J. A. Murphy

(C) 2016 Alajarin et al; licensee Beilstein-Institut. License and terms: see end of document.

\begin{abstract}
6-Phenylfulvenes bearing (1,3-dioxolan or dioxan)-2-yl substituents at ortho position convert into mixtures of 4- and 9-(hydroxy)alkoxy-substituted benz[f]indenes as result of cascade processes initiated by a thermally activated hydrogen shift. Structurally related fulvenes with non-cyclic acetalic units afforded mixtures of 4- and 9-alkoxybenz[f]indenes under similar thermal conditions. Mechanistic paths promoted by an initial [1,4]-, [1,5]-, [1,7]- or [1,9]-H shift are conceivable for explaining these conversions. Deuterium labelling experiments exclude the [1,4]-hydride shift as the first step. A computational study scrutinized the reaction channels of these tandem conversions starting by $[1,5]-,[1,7]-$ and $[1,9]-\mathrm{H}$ shifts, revealing that this first step is the rate-determining one and that the $[1,9]-\mathrm{H}$ shift is the one with the lowest energy barrier.
\end{abstract}

\section{Introduction}

Fulvenes (also known as pentafulvenes), [1-4] a unique class of trienes, have intrigued chemists for decades due to their theoretical interest [5,6] and synthetic applications [7-15]. In this latter sense, fulvenes can be involved in multiple modes of cyclization processes such as $[4+2][7,8],[6+2][9-11]$, and $[6+3]$ [12-15] cycloaddition reactions resulting in the construction of diverse fused ring systems. Other classical pericyclic processes that may potentially occur in fulvene fragments (electrocyclic and ene reactions, sigmatropic rearrangements and shifts) have received less attention, most probably with the only exception of the Claisen rearrangement [16]. Notably, thermally promoted $\mathrm{H}$-shifts remain, to the best of our knowledge, completely unexplored in fulvene frameworks $[17,18]$.

A part of our recent research focused on showing the special ability of cyclic acetalic functions (1,3-dioxolanes, thiolanes, 
oxathiolanes, dioxanes, dithianes, oxathianes) for promoting the migration of its acetalic $\mathrm{H}$ atom in a hydride-like manner. As result, we have disclosed a variety of tandem processes initiated by [1,5]- and [1,4]-hydride shifts from the acetalic carbon atom toward electrophilic molecular fragments [19-27]. Thus, we have reported that ortho-(1,3-dioxolan-2-yl)benzylidenemalonates 1 undergo tandem hydride shift/cyclization sequences leading to the corresponding indan-1-one-2,2-dicarboxylates 2 . Remarkably, the first step of these processes consists of an uncommon [1,4]-hydride shift of the acetalic $\mathrm{H}$ atom following the activation of the benzylidenemalonate fragment by scandium(III) triflate as the catalyst (Scheme 1) [27].

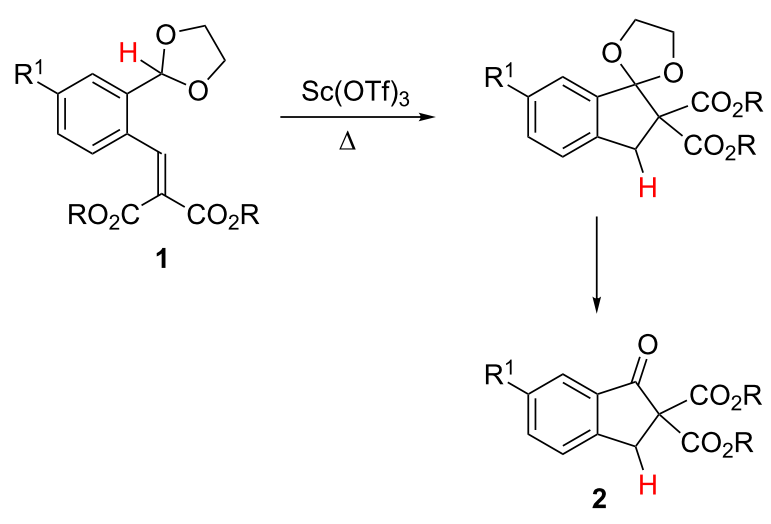

Scheme 1: Lewis acid-catalyzed [1,4]-H transfer/1,5-electrocyclization tandem processes of benzylidenemalonates 1 leading to indan-1-ones 2.

Because fulvenes are widely used as the direct precursors of cyclopentadienyl anions following the addition of nucleophiles, including the hydride anion, to its exocyclic $\mathrm{sp}^{2}$ carbon atom $[28,29]$, we wondered whether acetalic functions could be employed as internal $\mathrm{H}$ donors in intramolecular hydride-like shifts, analogous to that highlighted in Scheme 1, toward fulvene frameworks.

With this goal in mind we designed the unknown acetalfulvenes 3 (Scheme 2) as potential candidates for assaying the $[1,4]$-hydride shift of its acetalic $\mathrm{H}$ atom toward the exocyclic $\mathrm{C} 4$ carbon atom of the fulvene fragment (note the numbering in the Scheme 2). At this point it is worth noting that other possible $\mathrm{H}$ migrations were not ruled out at the outset of this investigation. For example, $\mathrm{C} 5$ and $\mathrm{C} 7$ may be the respective termini of sigmatropic [1,5]-H or [1,7]-H shifts, whereas $\mathrm{C} 6$ could be also prone to participate in a less common [1,9]-H shift [30-32]. The variety of potential intramolecular $\mathrm{H}$ migrations in these reactive species might well justify by itself the research here disclosed.

\section{Results and Discussion Experimental study}

The starting acetal-fulvenes 3 were prepared by the condensation of substituted 2-(1,3-dioxolan-2-yl)benzaldehydes 4 with cyclopentadiene following a well-established synthetic methodology [33]. With the aim of promoting the desired hydride transfer by thermal activation, we first heated the parent acetalfulvene 3a under a variety of reaction conditions (benzene $110^{\circ} \mathrm{C}$ sealed tube; toluene $120^{\circ} \mathrm{C}$ sealed tube; DMF $120^{\circ} \mathrm{C}$ ) but unfortunately without success. Only when a DMSO solution of $3 \mathbf{a}$ was heated at $120{ }^{\circ} \mathrm{C}$ for $7 \mathrm{~h}$ the acetal-fulvene converted into a complex mixture from which we were able to isolate the benz $[f]$ indenes $\mathbf{5 a}$ and $\mathbf{6 a}$, in a relative $2: 1$ ratio and a poor global yield (34\%). We next tested the same and similar processes in a microwave apparatus. As presumed, conversions of a series of acetal-fulvenes $\mathbf{3 a}-\mathbf{f}$ under $120 \mathrm{~W}$ microwave irradiation at $120{ }^{\circ} \mathrm{C}$ in DMSO required much shorter reaction times (20-40 $\mathrm{min})$ and led in all cases to the isolation of the respective benz[f]indenes 5 and $\mathbf{6}$, in a 2:1 ratio (Scheme 2). The overall yield of the isomeric mixtures $5+\mathbf{6}$ did not improve significantly with respect to the conventional thermal conditions previously used with 3a, ranging from medium to low as depicted in Table 1. Despite our chromatographic (column, thin-layer) efforts, additional pure products other than $\mathbf{5}$ and $\mathbf{6}$ could not be isolated from the complex final reaction mixtures.<smiles></smiles><smiles>[R]c1cc(C=C2C=CC=C2)c(C2OCCO2)cc1[R]</smiles><smiles>[R]c1cc2cc3c(c(O[R](=O)CO)c2cc1[R])CC=C3</smiles><smiles>[R]c1cc2cc3c(c(OCCO)c2cc1[R])C=CC3</smiles>

ii)

Scheme 2: Preparation of benz[f]indenes $\mathbf{5}$ and $\mathbf{6}$. Reagents and conditions: i) cyclopentadiene, pyrrolidine, anhydrous methanol, rt, $10 \mathrm{~h}$; ii) DMSO, microwave, $120^{\circ} \mathrm{C}, 120 \mathrm{~W}, 20-40 \mathrm{~min}$.

The structural determination of the reaction products, the 9- and 4-hydroxyalkoxy regioisomers $\mathbf{5}$ and $\mathbf{6}$, was easily accomplished by using the habitual analytical and spectroscopic techniques, whereas the distinction between each two regioisomers is basically supported by ${ }^{1} \mathrm{H}$ NMR NOE difference experiments. For the major isomers 5 enhancements of the signals due to the $\mathrm{H}$-C2 and $\mathrm{CH}_{2}$-OAr protons were observed when the methylenic protons were irradiated. In contrast, similar irradia- 


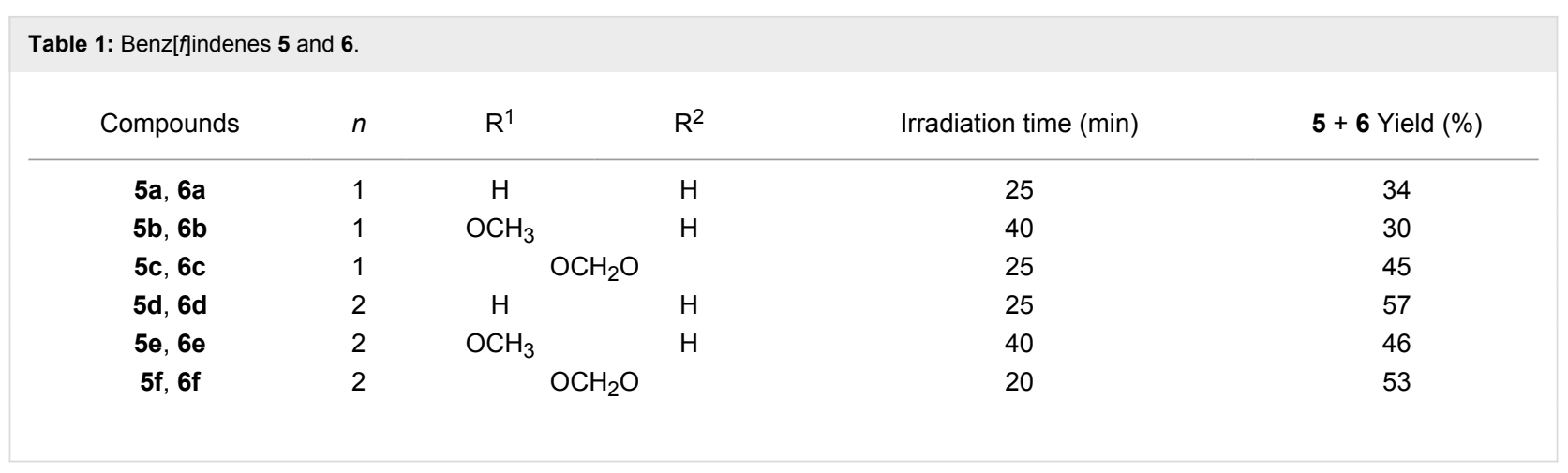

tion experiments with the minor isomers 6 revealed enhancements of $H$-C2 and $H$-C9.

As the $\mathrm{H}$ atom at the acetalic carbon of the initial fulvenes 3 apparently appears at the methylene group of both isomeric reaction products, it seems reasonable to hypothesize that the conversions $\mathbf{3} \rightarrow \mathbf{5}+\mathbf{6}$ are initiated by an $\mathrm{H}$ migration from the acetalic carbon toward the fulvene substructure. If this migration is a $[1,4]$-hydride shift to the exocyclic $\mathrm{C} 4$ carbon atom of the fulvene fragment, as initially postulated, this step (exemplified for the simplest fulvene 3a in Scheme 3) would lead to the dipolar intermediate $7 \mathbf{a}$ which reasonably would undergo further cyclization to 8a thus building the benz[f]indene substructure present in the final reaction products. For the conversion of intermediate $\mathbf{8 a}$ into the final mixture of $\mathbf{5 a}$ and 6a several mechanistic paths are conceivable, each one involving additional $\mathrm{H}$ shifts and a fragmentation step, the opening of the dioxolane ring by a formal $\beta$-elimination reaction.
A number of additional mechanistic alternatives arise by considering that the experimentally observed transformations of 3 are initiated by other $\mathrm{H}$ shifts alternative to the initially expected $[1,4]$ one. Thus, a [1,5]-H shift from the acetalic carbon of $3 \mathbf{a}$ to the $\mathrm{C} 5$ carbon atom of the cyclopentadiene ring, would lead to the transient ortho-quinodimethane structure 9a, which might transform into two similar intermediates, 10a and 11a, by a sequence of consecutive [1,5]-H shifts around the cyclopentadiene ring. Next, intermediates $10 \mathrm{a}$ and 11a would undergo $6 \pi$-electrocyclic ring closures $(6 \pi$-ERC) to the respective dihydrobenzindenes 12a and 13a. Finally, these two species would experiment the acetalic ring opening by a formal $\beta$-elimination proccess leading to the respective final products $5 \mathbf{a}$ and $\mathbf{6 a}$ (Scheme 4).

This mechanistic scheme is further complicated when considering that ortho-quinodimethane intermediates 10a and 11a could also result from the respective [1,9]-H and [1,7]-H shifts<smiles>C1=CC(=Cc2ccccc2C2OCCO2)C=C1</smiles><smiles>CCCC</smiles><smiles></smiles>

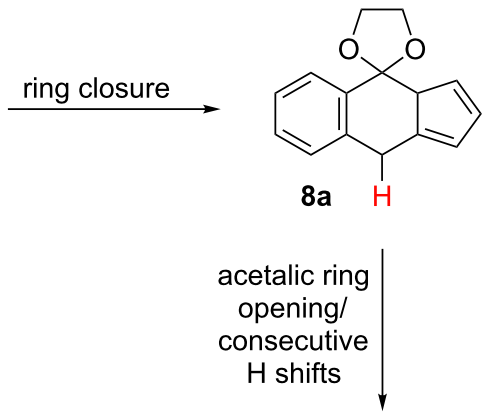<smiles>OCCOc1c2c(cc3ccccc13)C=CC2</smiles><smiles>OCCOc1c2c(cc3ccccc13)CC=C2</smiles> 


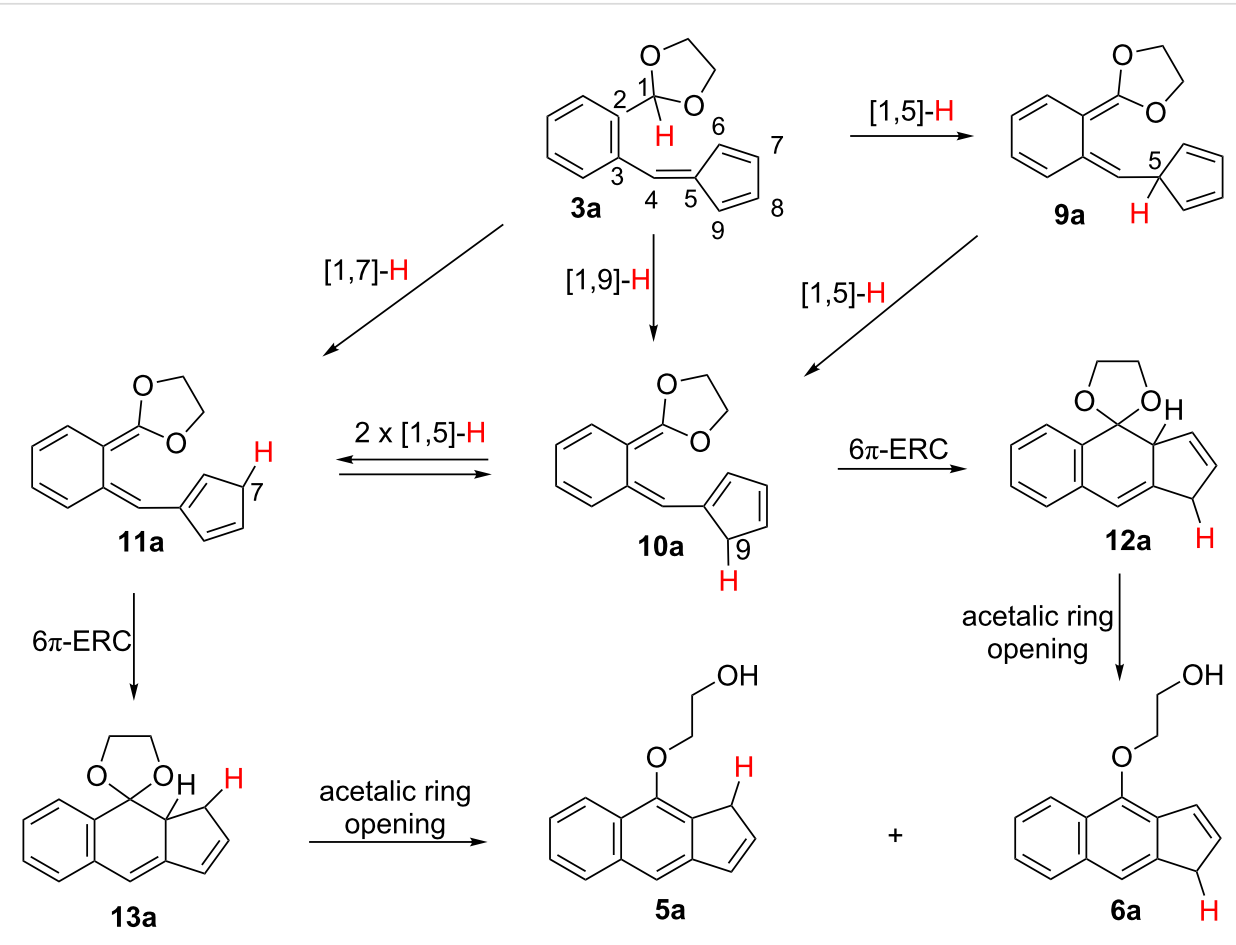

Scheme 4: Alternative mechanistic paths for the conversion $\mathbf{3 a} \rightarrow \mathbf{5 a}+\mathbf{6} \mathbf{a}$ initiated by $[1,5]-,[1,7]-$ or $[1,9]-\mathbf{H}$ shifts.

occurring in the starting acetal-fulvene 3a (Scheme 4). Thus, the different mechanistic paths represented in Scheme 4 for explaining the conversion $\mathbf{3 a} \rightarrow \mathbf{5 a}+\mathbf{6 a}$ share several common steps, essentially differing in their first hydrogen shift, $[1,5]-$, [1,7]- or [1,9]-H.

Obviously, any attempt to discern which one is the actual reaction path (if only one!) among the range of potential mechanistic alternatives for these transformations seems a huge task. Seeking for additional experimental data in order to approach such objective, we reasoned that the mechanism initiated by a $[1,4]$-hydride shift, as summarized in Scheme 3, could be differentiated from those starting by [1,5]-, [1,7]- or [1,9]-H shifts, represented in Scheme 4, by deuterium labelling experiments. Thus, if the conversion of the deuterated acetal-fulvene 14, in which deuterium replaces the proton at the acetalic carbon of 3a, was actually initiated by a $[1,4]$-deuteride shift, the transformation of the dihydrobenzo[f]indenic species $\mathbf{1 5}$, which should form in first instance, would yield the final benz[f]indenes via intermediates $\mathbf{1 6}$ and $\mathbf{1 7}$. These are labelled with deuterium at $\mathrm{C} 4$ of the major product 18 and at C9 of the minor one 19 as well as probably at additional positions of the fused five-membered ring (Scheme 5).

With this in mind we prepared the monodeuterated acetalfulvene 14 (see Supporting Information File 1) and submitted it to the habitual reaction conditions. As result, a mixture of the monodeuterated benz[f]indenes $\mathbf{1 8}$ and $\mathbf{1 9}$ was obtained, again in a relative 2:1 ratio (Scheme 6). ${ }^{1} \mathrm{H}$ NMR analyses showed that only protons, not deuteriums, were linked to the $\mathrm{C} 4$ atom of 18 and to $C 9$ of 19. Instead, one deuterium atom is found at the methylene group of each regioisomer.

Moreover, we carried out a second labelling experiment by using now as starting material the monodeuterated acetalfulvene $\mathbf{2 0}$ bearing the deuterium atom at $\mathrm{C} 4$. This species converted into a 2:1 mixture of the monodeuterated regioisomers 21 and 22 as the only isolated reaction products (Scheme 6). In both compounds, the deuteration percentage at their respective $\mathrm{C} 4$ and $\mathrm{C} 9$ positions was determined, by ${ }^{1} \mathrm{H}$ NMR analyses, to be higher than $98 \%$.

This latter result shows that the deuterium atom attached at $\mathrm{C} 4$ in the original acetal-fulvene does not migrate in the course of the reaction. In combination, these two labelling experiments are conclusive for discarding an initial [1,4]-deuteride (or hydride) shift as the first step of the previously discussed conversions since, in such a case, we should have found deuterium linked to $\mathrm{C} 4 / \mathrm{C} 9$ of the benzindenes $\mathbf{1 8} / \mathbf{1 9}$ resulting from the first labelling experiment.

Next we tried to understand why the regioisomeric benz $[f]$ indenes were, in all our reactions, produced in a ratio close to $2: 1$. It is well known that $1 H$-indenes are prone to 

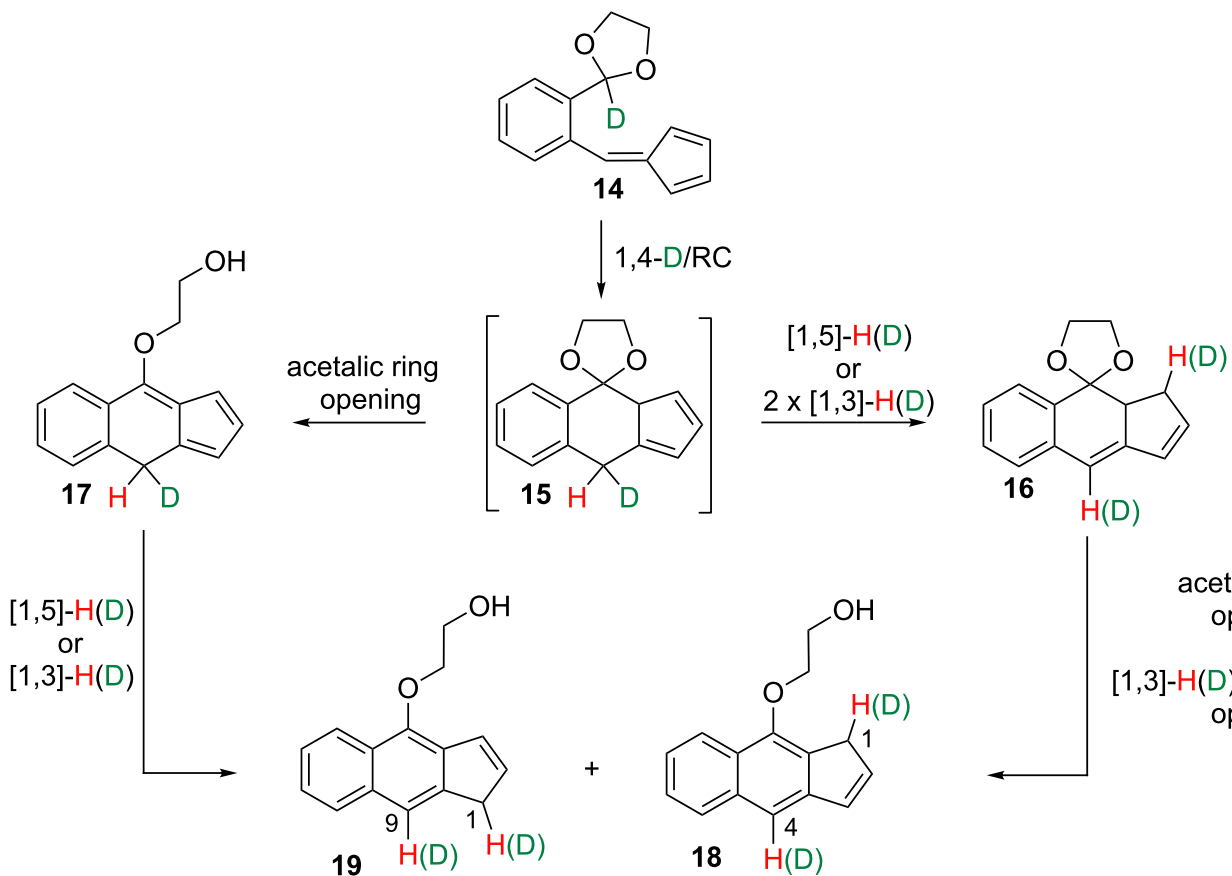

acetalic ring opening or

$[1,3]-H(D) / a c e t a l i c$ ring opening<smiles>O=C(O)C1(O)OCCO1</smiles>

i)<smiles></smiles><smiles>OCCOc1cc2ccccc2c2c1C=CC2=[18O]</smiles><smiles>[18O]=C(c1ccccc1)c1ccccc1C1OCCO1</smiles>

20

i)<smiles>OCCOc1c2c(c([18OH])c3ccccc13)CCC2</smiles>

21<smiles>OCCOc1c2c(c([18O])c3ccccc13)CCC2</smiles>

22

Scheme 6: Preparation of deuterated benz[f]indenes $18+19$ and $21+22$. Reagents and conditions: i) DMSO, microwave, $120{ }^{\circ} \mathrm{C}, 120 \mathrm{~W}, 40$ min.

undergo isomerization by $\mathrm{H}$ or group migrations at its cyclopentadiene ring [34-47]. Consequently, we postulated that such an isomeric ratio would correspond to the thermodynamic equilibrium between both isomers, $\mathbf{5}$ and $\mathbf{6}$, established by two consecutive $[1,5]-\mathrm{H}$ shifts around its five-membered ring. To test this hypothesis, we heated a $4: 1$ mixture of isomeric $5 \mathbf{c}$ and $\mathbf{6 c}$ in deuterated DMSO solution at $120^{\circ} \mathrm{C}$ for $24 \mathrm{~h}$. In this way we could verify by ${ }^{1} \mathrm{H}$ NMR analyses of reaction aliquots that the initial isomeric ratio remained constant over time and heating. Interestingly, the initial 4:1 ratio changed to $2: 1$ at the end of a related experiment carried out by stirring a DMSO solution of the same isomeric mixture in the presence of a catalytic amount of triethylamine at room temperature for $2 \mathrm{~h}$ (Scheme 7).

This result seems to indicate that the experimentally recurrent 2:1 proportion between the regioisomeric products 5 and $\mathbf{6}$, 

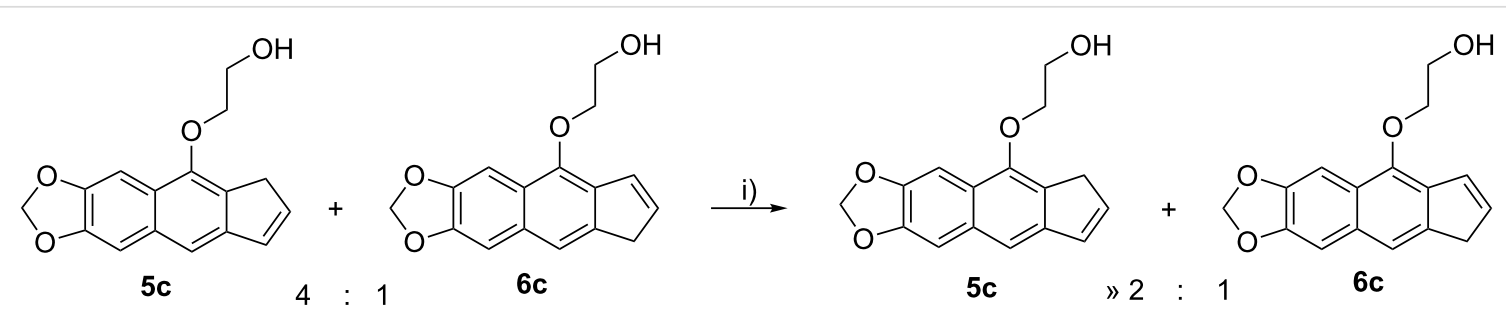

Scheme 7: Reagents and conditions: i) triethylamine (10\%), DMSO, rt, $2 \mathrm{~h}$

reached by equilibration in the latter experiment, should be due to the presence of adventitious minor amounts of basic species either in the DMSO solutions of the experiments or in the course of the processing of the crude reaction mixtures and the purification steps.

Besides, we also explored the thermally induced transformations of related fulvenes bearing non-cyclic acetalic units (Scheme 8 and Table 2). To this end, benzaldehydes $\mathbf{2 3}$ were transformed into fulvenes $\mathbf{2 4}$ by the usual procedure, whereas its microwave heating (DMSO, $120^{\circ} \mathrm{C}, 120 \mathrm{~W}$ ) yielded a mixture of the benz[f]indenes $\mathbf{2 5}$ and $\mathbf{2 6}$ in the habitual 2:1 ratio. These conversions most probably occur, in mechanistic terms, similarly to those of the acetal-fulvenes 3 (Scheme 4), although in the present cases with the formal $\beta$-elimination of a methanol or ethanol molecule.

These results show that non-cyclic acetalic units are as effective as the cyclic ones on achieving the conversion of acetalfulvenes into the corresponding benz $[f]$ indenes under microwave irradiation.

\section{Computational study}

With the aim of scrutinizing the putative reaction paths leading from the fulvene $\mathbf{3 a}$ to the isomeric benz[f]indenes $\mathbf{5 a}$ and $\mathbf{6 a}$ we have carried out a computational study at the B3LYP/6$31+\mathrm{G}^{* *}$ theoretical level. Scheme 9 shows the diversity of the computed reaction paths leading from reactants to products. The geometries of the located transition structures associated to the first mechanistic step of each path, the $\mathrm{H}$ shift, are shown in Figure 1 [48].

We anticipated three general reaction channels, paths A-C (see Scheme 9). In fact, these pathways only differ in the first step. Path A starts by a [1,5]-H shift, path B by a [1,9]-H shift and path $\mathrm{C}$ by a $[1,7]-\mathrm{H}$ shift. This overall mechanistic scheme is somewhat complicated due to the number of steps of each reaction path and by the fact that some stationary points belong to more than one of these three pathways. Obviously we also envisaged a fourth mechanistic alternative, path $\mathrm{D}$, just that initially conceived starting by the $[1,4]$-hydride shift of the acetalic $\mathrm{H}$ atom to the exocyclic $\mathrm{C} 4$ carbon atom of the fulvene unit. All our efforts aimed to locate its corresponding transition<smiles>[R1]OC([R10])c1cc([R])c([R])cc1C=O</smiles>

23<smiles>[R1]OC([R2])c1cc([R])c([R])cc1C=C1C=CC=C1</smiles>

24<smiles>[C+]1=CC=CC1</smiles><smiles>[Z10]Oc1c2c(cc3cc([R])c([R])cc13)C=CC2</smiles>

25<smiles>[R1]Oc1c2c(cc3cc([R])c([R])cc13)CC=C2</smiles>

26

Scheme 8: Preparation of benz[f]indenes $\mathbf{2 5}$ and 26. Reagents and conditions: i) cyclopentadiene, pyrrolidine, anhydrous methanol, rt, $10 \mathrm{~h}$; ii) DMSO, microwave, $120^{\circ} \mathrm{C}, 120 \mathrm{~W}, 20-40 \mathrm{~min}$.

Table 2: Acetal-fulvenes 24 and benz[ $f$ jindenes $\mathbf{2 5}$ and 26 .

\begin{tabular}{|c|c|c|c|c|c|c|}
\hline Compounds & $\mathrm{R}^{1}$ & $\mathrm{R}^{2}$ & $\mathrm{R}^{3}$ & $\begin{array}{c}24 \\
\text { Yield (\%) }\end{array}$ & $\begin{array}{l}\text { Irradiation } \\
\text { time (min) }\end{array}$ & $\begin{array}{c}25+26 \\
\text { Yield (\%) }\end{array}$ \\
\hline $24 a-26 a$ & $\mathrm{H}$ & $\mathrm{H}$ & $\mathrm{CH}_{3}$ & 68 & 10 & 54 \\
\hline $24 b-26 b$ & $\mathrm{OCH}_{3}$ & $\mathrm{H}$ & $\mathrm{CH}_{3}$ & 71 & 15 & 42 \\
\hline $24 c-26 c$ & \multicolumn{2}{|c|}{$\mathrm{OCH}_{2} \mathrm{O}$} & $\mathrm{CH}_{3}$ & 81 & 15 & 47 \\
\hline $24 d-26 d$ & $\mathrm{H}$ & $\mathrm{H}$ & $\mathrm{CH}_{3} \mathrm{CH}_{2}$ & 74 & 15 & 60 \\
\hline
\end{tabular}



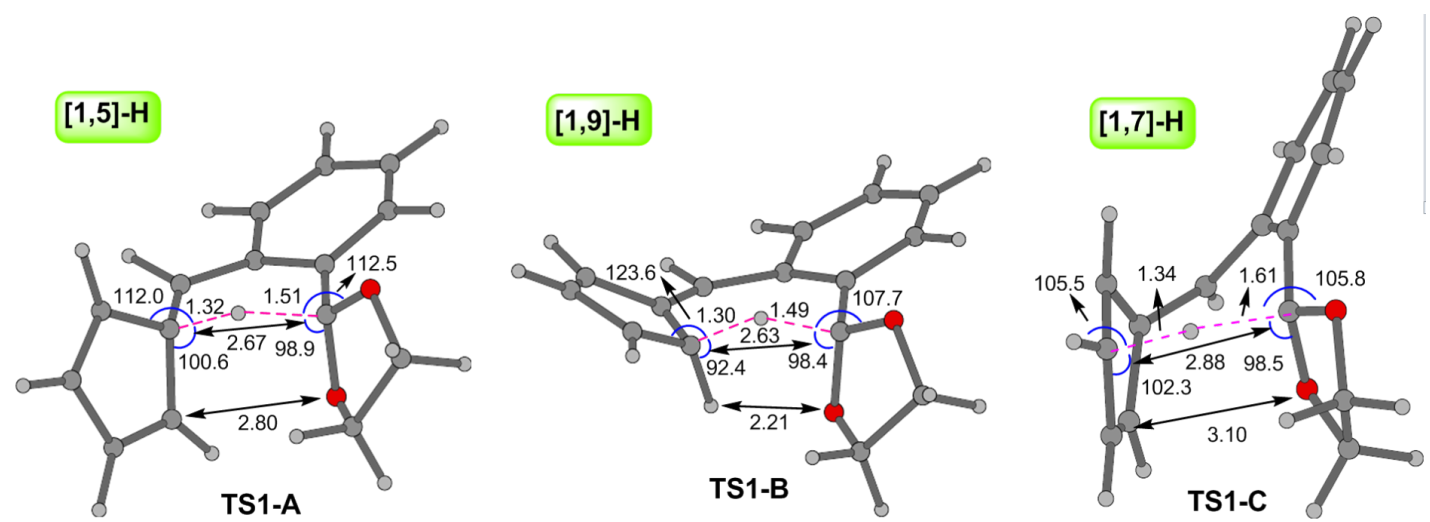

Figure 1: Optimized geometry of transition structures TS1-A, TS1-B, and TS1-C computed at the B3LYP/6-31+G** theoretical level. Distances in Ångstroms, bond angles in degrees.<smiles></smiles>

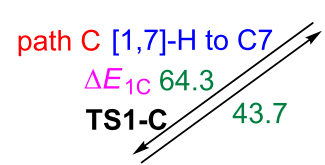

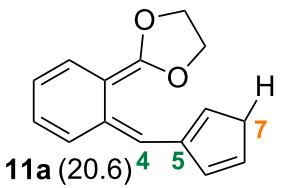

\section{$[1,5]-\mathrm{H}$ to $\mathrm{C} 7$} TS5<smiles>C=C[PbH2]</smiles>
TS6
$\Delta E_{6} 13.9$|| $\begin{aligned} & 6 \pi-E R C \\ & 52.9\end{aligned}$<smiles></smiles>
13a $(-18.4)$

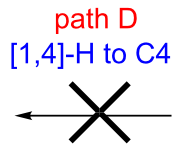<smiles>C1=CCC(C=Cc2ccccc2C2OCCO2)=C1</smiles>

path $B$ [1,9]-H to C9 $\Delta E_{1 \mathrm{~B}} 41.4 \mid 22.7$

TS1-B

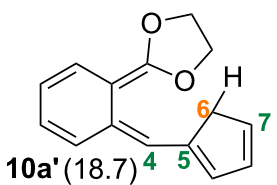

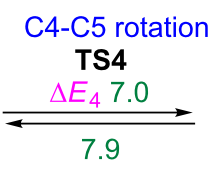

[1,5]-H to $\mathrm{C} 6$ $\Delta E_{3} 19.0$

TS3<smiles></smiles>
$10 a^{\prime}(18.7)$<smiles>OCCOc1c2c(cc3ccccc13)CC=C2</smiles>
acetalic ring<smiles></smiles>

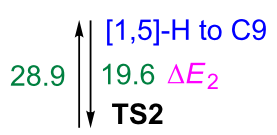<smiles>C1=CCC(C=c2ccccc2=C2OCCO2)=C1</smiles>
$6 \pi$-ERC $\uparrow \mid$ TS7 $47.2 \downarrow 17.0 \Delta E_{7}$<smiles></smiles>

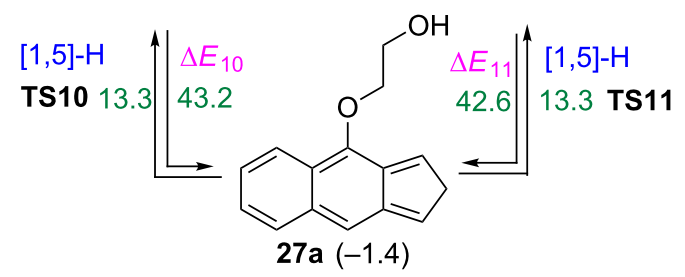

Scheme 9: Mechanistic paths for the conversion of fulvene $\mathbf{3 a}$ into the benz $[f]$ indenes $\mathbf{5 a}$ and $\mathbf{6 a}$ showing the energy barriers of each step in $\mathrm{kcal} \cdot \mathrm{mol}^{-1}$ as computed at the B3LYP/6-31+G $\mathrm{G}^{* *}$ theoretical level (between parentheses the relative electronic energies of the minima in $\mathrm{kcal} \cdot \mathrm{mol}^{-1}$ ).

structure were unsuccessful. Nevertheless, this latter mechanistic alternative was discarded by the isotopic labelling experiments commented above.
In the following paragraphs we intend to discuss in a simplified way the results of our calculations on the potential surface of the transformations summarized in Scheme 9. 
The first step of path A consists of a [1,5]-H shift from the acetalic carbon atom to C5 (see the numbering in Scheme 9). We located the transition structure TS1-A, connecting the fulvene 3a with the ortho-quinodimethane intermediate 9a. As expected TS1-A shows the typical geometry of a suprafacial hydrogen shift (see Figure 1), the computed energy barrier associated to this step being fairly high, $47.5 \mathrm{kcal} \cdot \mathrm{mol}^{-1}$. Intermediate 9a could then experiment two alternative [1,5]-H shifts of its $\mathrm{H}-\mathrm{C} 5$ proton, migrating either to $\mathrm{C} 6$ or to $\mathrm{C} 9$, its two vicinal carbon atoms at the cyclopentadiene ring. For the $[1,5]-\mathrm{H}$ shift to $\mathrm{C} 9$ we located the transition structure TS2, $19.6 \mathrm{kcal} \cdot \mathrm{mol}^{-1}$ above in energy than 9a, connecting it with its isomer 10a. For the alternative $[1,5]-\mathrm{H}$ shift to $\mathrm{C} 6$ we located the transition structure TS3, $19.0 \mathrm{kcal} \cdot \mathrm{mol}^{-1}$ above in energy than 9a, leading to the isomeric structure $\mathbf{1 0 a}$ ' which is in fact a rotamer of $\mathbf{1 0 a}$. These two energy barriers are reasonably low and should be easily surmountable under the experimental reaction conditions. Additionally, we were able to locate a transition structure, TS4, connecting 10a and 10a' by rotation around the $\mathrm{C} 4-\mathrm{C} 5$ single bond. The computed barrier for the conversion of $\mathbf{1 0}$ into $\mathbf{1 0 a}$ ' via TS4 is only $7.0 \mathrm{kcal} \cdot \mathrm{mol}^{-1}$, whereas the one for the reverse transformation is $7.9 \mathrm{kcal} \cdot \mathrm{mol}^{-1}$. Accordingly, equilibration between $10 a$ and $\mathbf{1 0 a}$ ' is predicted to occur rapidly by $\mathrm{C} 4-\mathrm{C} 5$ bond rotation rather than by two consecutive $[1,5]-\mathrm{H}$ shifts via the isomeric intermediate 9a (see Scheme 9).

Intermediate 10a' can also convert into a third ortho-quinodimethane isomer 11a via the transition structure TS5 by another [1,5]-H shift from $\mathrm{C} 6$ to its vicinal $\mathrm{C} 7$ carbon atom at the cyclopentadiene ring. The computed energy barrier for this step is $27.6 \mathrm{kcal} \cdot \mathrm{mol}^{-1}$, significantly higher than those corresponding to the similar [1,5]-H shifts via TS2 and TS3 commented above $\left(19.6\right.$ and $\left.19.0 \mathrm{kcal} \cdot \mathrm{mol}^{-1}\right)$. The lower barriers of these two latter transition structures are attributable to its more extended conjugation in comparison with the partially crossconjugated TS5.

A series of reaction steps starting from intermediates 10a and 11a can lead respectively to the final benzindenes $5 \mathbf{a}$ and $\mathbf{6 a}$. Thus, intermediate 11a undergoes a disrotatory $6 \pi$-electrocyclic ring closure [49] via the transition structure TS6 to give the tricyclic species 13a. By an analogous electrocyclic ring closure through TS7, compound 10a is converted into the isomeric spirotricycle 12a. The computed energy barriers for these processes are relatively small, 13.9 and $17.0 \mathrm{kcal} \cdot \mathrm{mol}^{-1}$ respectively. Again the differences in the extent of the electronic conjugation in these electrocyclization transition states can give account of the relative stabilities of TS6 and TS7.

Two transition structures, TS8 and TS9 were located for the respective transformations of 13a and 12a into the final benzin- denes $5 \mathbf{a}$ and $\mathbf{6 a}$, involving each one the opening of the acetalic ring with simultaneous transfer of an hydrogen to one of the oxygen atoms (in other words, a concerted $\beta$-elimination along a $\mathrm{C}-\mathrm{C}$ single bond), with the concomitant aromatization of the central ring. The computed energy barriers for these concerted $\beta$-eliminations are high, 41.7 and $40.8 \mathrm{kcal} \cdot \mathrm{mol}^{-1}$ respectively $[50]$.

Concerning the alternative reaction paths $\mathrm{B}$ and $\mathrm{C}$, we have located essentially the same stationary points that in path A with the sole difference of the respective first mechanistic steps. Path B starts with a [1,9]-H sigmatropic rearrangement through TS1B leading to intermediate 10a', which then transforms via the mechanistic paths commented above. The geometry of TS1-B (see Figure 1) is in accordance with a suprafacial transfer of the $\mathrm{H}$ atom between the acetalic carbon and $\mathrm{C} 6$. The calculated energy barrier associated to this step is $41.4 \mathrm{kcal} \cdot \mathrm{mol}^{-1}$, $6.1 \mathrm{kcal} \cdot \mathrm{mol}^{-1}$ lower in energy than the initial [1,5]-H shift of path A. This difference could be rationalized attending to the geometries of both transition structures TS1-A and TS1-B, more specifically to the distance between the two carbon atom termini of the H migration, shorter in TS1-B (2.63 $\AA)$ than in TS1-A (2.67 $\AA)$. Therefore, TS1-B is earlier than TS1-A. The geometry of TS1-B also accounts for its greater conjugation as the spatial positioning of the cyclopentadiene ring allows its orbital overlapping with the rest of the $\pi$ system. Moreover, TS1-B is less sterically congested and also less distorted than TS1-A (see the bond distances and bond angles displayed in Figure 1).

For the first step of path $\mathrm{C}$ we have located a transition structure, TS1-C, connecting fulvene 3a with the intermediate 11a by a $[1,7]-\mathrm{H}$ sigmatropic shift (see Scheme 9). The computed energy barrier is very high, $64.3 \mathrm{kcal} \cdot \mathrm{mol}^{-1}$. This large value is probably due to the heptatrienic fragment not being able of adopting the helical all s-cis conformation, optimal for an antarafacial $[1,7]-\mathrm{H}$ shift, as result of the conformational restrictions imposed by the cyclopentadiene ring (Figure 1). As a consequence, the distance between the two carbon atom termini of the $\mathrm{H}$ migration is considerably long ( $2.88 \AA)$, thus accounting for the high computed energy barrier.

To summarize so far, by comparing the energy barriers associated to the three alternative $\mathrm{H}$ shifts, this study predicts that path $\mathrm{B}$ is the one involving the lowest energy barrier and, in accordance, the calculations predict that the transformation of fulvene 3a into the benzindenes 5a and 6a should take place via an initial [1,9]-H shift.

Moreover, we also considered that $\mathbf{5 a}$ and $\mathbf{6 a}$ could equilibrate by two consecutive $[1,5]-\mathrm{H}$ shifts occurring at the five-mem- 
bered ring. By exploring the potential energy surface associated to these transformations we were able to locate transition structures TS10 and TS11 connecting 5a and 6a through the intermediate 27a (see Scheme 9). The computed energy barriers for the conversions $5 \mathbf{a} \rightarrow \mathbf{2 7} \mathbf{a}$, and $\mathbf{6 a} \rightarrow \mathbf{2 7} \mathbf{a}$ are fairly high, 43.2 and $42.6 \mathrm{kcal} \cdot \mathrm{mol}^{-1}$, respectively, as expected on going from a fully aromatic central ring to an orthoquinoid structure, whereas those calculated for the reverse conversions are considerably lower $\left(13.3 \mathrm{kcal} \cdot \mathrm{mol}^{-1}\right.$ in both cases).

By analysing the overall picture showing the different mechanistic paths connecting $3 \mathbf{a}$ with the final benzindenes $5 \mathbf{a}$ and $\mathbf{6 a}$ we can extract the following conclusions:

1) On going from $\mathbf{3 a}$ to the two tricyclic intermediates $\mathbf{1 2 a}$ and 13a, the rate determining reaction step is predicted to be the first one, i.e. the initial hydrogen migration, and this study predicts that a $[1,9]-\mathrm{H}$ shift is less costly in terms of energy than a $[1,5]-\mathrm{H}$ or $[1,7]-\mathrm{H}$ one. The more extended conjugation, the lower steric hindrance and the shorter $\mathrm{C}-\mathrm{C}$ distance between the two carbon atoms termini of the H migration in TS1-B, the transition structure of the [1,9]-H shift, can account for its lower energy when compared with those of the alternative two other $\mathrm{H}$ shifts. Nevertheless, the higher electronic conjugation in TS1-B, in comparison with those of TS1-A and TS1-C, could be also decisive in accounting for the differences in the respective energy barriers.

2) Concerning the two key polyenes 10a and 11a, precursors of the tricycles 12a and 13a, respectively, the computed energy barriers of this study show that i) $\mathbf{1 0 a}$ most probably forms by an easy rotational isomerization of $\mathbf{1 0 a}$, instead of the alternative path involving the $[1,5]-\mathrm{H}$ shift from 9a; and ii) 11a will form mainly from 10a' rather than directly from 3a. That is, 10a and 11a should form via intermediate 10a' resulting from the $[1,9]-\mathrm{H}$ shift, which then transforms into 11a by a $[1,5]-\mathrm{H}$ shift (barrier of $27.6 \mathrm{kcal} \cdot \mathrm{mol}^{-1}$ ) or equilibrates to its rotamer 10a (barrier of $7.0 \mathrm{kcal} \cdot \mathrm{mol}^{-1}$ ).

3) The two $6 \pi$-electrocyclic ring closures converting respectively 10a and 11a into 12a and 13a involve low energy barriers, that corresponding to the conversion of 11a into 13a being lower than that of $10 \mathrm{a}$ into $12 \mathrm{a}\left(13.9\right.$ and $17.0 \mathrm{kcal} \cdot \mathrm{mol}^{-1}$, respectively).

4) The overall processes $\mathbf{3 a} \rightarrow \mathbf{5 a}$ and $\mathbf{3 a} \rightarrow \mathbf{6 a}$ are exothermic by 31.3 and $30.7 \mathrm{kcal} \cdot \mathrm{mol}^{-1}$, respectively. The interconversion between $\mathbf{5 a}$ and $\mathbf{6 a}$ is predicted to take place via two consecutive [1,5]-H shifts at the pentagonal ring through transient intermediate $27 \mathbf{a}$.
As a final point, we have also explored the potential energy surface associated to these conversions by considering the effect of the solvent used in the experimental study, DMSO. The computed energy barriers in the gas phase and in DMSO are depicted in Table 3.

\begin{tabular}{|c|c|c|}
\hline $3 a \rightarrow 5 a+6 a$ & Gas & DMSO \\
\hline$\Delta E_{1 \mathrm{~A}}$ & 47.5 & 47.3 \\
\hline$\Delta E_{2}$ & 19.6 & 19.0 \\
\hline$\Delta E_{3}$ & 19.0 & 17.6 \\
\hline$\Delta E_{1 \mathrm{~B}}$ & 41.4 & 39.9 \\
\hline$\Delta E_{4}$ & 7.0 & 6.9 \\
\hline$\Delta E_{5}$ & 27.6 & 26.6 \\
\hline$\Delta E_{1 \mathrm{C}}$ & 64.3 & 63.6 \\
\hline$\Delta E_{6}$ & 13.9 & 14.4 \\
\hline$\Delta E_{7}$ & 17.0 & 17.5 \\
\hline$\Delta E_{8}$ & 41.7 & 36.8 \\
\hline$\Delta E_{9}$ & 40.8 & 37.3 \\
\hline$\Delta E_{10}$ & 43.2 & 42.3 \\
\hline$\Delta E_{11}$ & 42.6 & 41.4 \\
\hline
\end{tabular}

asee Scheme 9 for the notation of the energy barriers.

In general, the values of the energy barriers do not vary noticeably in DMSO when compared with those in gas phase. Only $\Delta E_{8}$ and $\Delta E_{9}$ are appreciably lower in DMSO with respect to those in gas phase by 3.9 and $3.5 \mathrm{kcal} \cdot \mathrm{mol}^{-1}$, respectively (Table 3). Consequently, according to these calculations, the rate determining step in the transformations $\mathbf{3 a} \rightarrow \mathbf{5 a}$ and $\mathbf{3 a} \rightarrow \mathbf{6 a}$ in DMSO should be the first one, i.e. the [1,9]-H shift, with an energy barrier slightly lower than that calculated in the gas phase.

In summary, this computational study shows that the conversion of fulvene 3a into the benzindenes 5a and 6a could take place by a variety of alternative reaction paths according to a complicated mechanistic scheme. By analysing in detail the energy barriers computed for each mechanistic step, the energetically preferred path starts with a [1,9]-H sigmatropic rearrangement of the acetalic hydrogen atom leading to an orthoquinodimethane intermediate, further transforming into the isomeric final products by two alternative reaction channels. These two latter pathways may involve up to three consecutive steps such as $[1,5]-\mathrm{H}$ shifts, $6 \pi$-electrocyclic ring closures, $\mathrm{C}-\mathrm{C}$ rotations and formal $\beta$-eliminations. The interconversion between the isomeric benzindenes 5a and 6a could also occur by means of two consecutive [1,5]-H shifts through an unstable benzisoindene intermediate. 


\section{Conclusion}

The ability of benzofulvenes bearing 1,3-dioxolane or -dioxane units in ortho position for undergoing cascade processes initiated by an H shift step has been tested. Such acetal-fulvenes, under thermal activation, transformed into mixtures of the corresponding 4- and 9-(hydroxy)alkoxy-substituted benz $[f]$ indenes in a 1:2 ratio. Analogous fulvenes bearing noncyclic dialkoxymethyl units when submitted to similar thermal conditions also afforded 1:2 mixtures of the respective 4 and 9-alkoxybenz $[f]$ indenes. Such 1:2 ratio has been interpreted as the one corresponding to the thermodynamic equilibrium established between both isomers. Mechanistic paths initiated by an initial [1,4]-, [1,5]-, [1,7]- or [1,9]-H shift are conceivable for explaining these cascade transformations leading to benz[f]indenes. The results of deuterium labelling experiments excluded a [1,4]-hydride shift as the initial step. The reaction of the unsubstituted 1,3-dioxolane-fulvene has been computationally studied by DFT methods. The results of this study revealed that the first mechanistic step, the $\mathrm{H}$ shift, is the rate-determining one and that, among the alternative $[1,5]-,[1,7]-$ or $[1,9]-\mathrm{H}$ migrations, the energy barrier of the $[1,9]-\mathrm{H}$ shift is the lowest one, a fact that is rationalised attending to some key structural and electronic characteristics of the respective transition states. The calculations have also shown that the tandem conversions of the starting fulvenes into benz $[f]$ indenes are exergonics, the 9-substituted regioisomer being the thermodynamically-controlled major product, in accordance with the experimental results.

\section{Supporting Information}

\section{Supporting Information File 1 \\ Experimental part. \\ [http://www.beilstein-journals.org/bjoc/content/ \\ supplementary/1860-5397-12-28-S1.pdf]}

\section{Supporting Information File 2}

Computational part.

[http://www.beilstein-journals.org/bjoc/content/ supplementary/1860-5397-12-28-S2.pdf]

\section{Acknowledgements}

This work was supported by the Ministerio de Economía y Competitividad of Spain (Project N ${ }^{\circ}$ CTQ2014-56887-P) and the Fundación Séneca-CARM (Project No 19240/PI/14).

\section{References}

1. Neuenschwander, M. Helv. Chim. Acta 2015, 98, 731-762. doi:10.1002/hlca.201400210
2. Neuenschwander, M. In Double-Bonded Functional Groups; Patai, S., Ed.; John Wiley \& Sons: Chichester, 1989; Vol. 2, pp 1131-1286. doi:10.1002/9780470772256.ch4

3. Bergmann, E. D. Chem. Rev. 1968, 68, 41-84. doi:10.1021/cr60251a002

4. Day, J. H. Chem. Rev. 1953, 53, 167-189. doi:10.1021/cr60165a002

5. Dahlstrand, C.; Rosenberg, M.; Kilsa, K.; Ottosson, $\mathrm{H}$. J. Phys. Chem. A 2012, 116, 5008-5017. doi:10.1021/jp3032397

6. Oziminsky, W. P.; Krygowski, T. M.; Fowler, P. W.; Soncini, A. Org. Lett. 2010, 12, 4880-4883. doi:10.1021/ol102037e

7. Hong, B.-C.; Chen, F.-L.; Chen, S.-H.; Liao, J.-H.; Lee, G.-H. Org. Lett. 2005, 7, 557-560. doi:10.1021/ol047730m

8. Gleiter, R.; Borzyk, O. Angew. Chem., Int. Ed. Engl. 1995, 34, 1001-1003. doi:10.1002/anie.199510011 And references therein.

9. Hong, B.-C.; Shr, Y.-J.; Wu, J.-L.; Gupta, A. K.; Lin, K.-J. Org. Lett. 2002, 4, 2249-2252. doi:10.1021/ol026103z

10. Wu, T. C.; Houk, K. N. J. Am. Chem. Soc. 1985, 107, 5308-5309. doi:10.1021/ja00304a065

11. Hafner, K.; Suda, M. Angew. Chem., Int. Ed. Engl. 1976, 15, 314-315. doi:10.1002/anie.197603141

12. He, Z.-L.; Teng, H.-L.; Wang, C.-J. Angew. Chem., Int. Ed. 2013, 52, 2934-2938. doi:10.1002/anie.201208799

13. Potowski, M.; Antonchick, A. P.; Waldmann, H. Chem. Commun. 2013, 49, 7800-7802. doi:10.1039/c3cc43824d

14. Hong, B.-C.; Gupta, A. K.; Wu, M.-F.; Liao, J.-H.; Lee, G.-H. Org. Lett. 2003, 5, 1689-1692. doi:10.1021/ol034329b

15. Barluenga, J.; Martinez, S.; Suárez-Sobrino, A. L.; Tomás, M. J. Am. Chem. Soc. 2001, 123, 11113-11114. doi:10.1021/ja011600g

16. Olsson, T.; Wennerstroem, O. Acta Chem. Scand., Ser. B 1979, B33, 256-260.

17. Prinzbach, V. H.; Rosswog, W. Tetrahedron Lett. 1963, 4, 1217-1221. doi:10.1016/S0040-4039(01)90806-8

See for a base-catalyzed [1,5]-H shift in cycloheptatrienyl-substituted fulvenes.

18. Van de Ven, L. J. M.; Keulemans-Lebbink, J. L. M.; de Haan, J. W.; Kloosterziel, H. J. Chem. Soc. D 1970, 1509a. doi:10.1039/c2970001509a

See for the preparation of a fulvene via a photochemical $[1,7]-\mathrm{H}$ shift

19. Alajarin, M.; Bonillo, B.; Ortin, M.-M.; Sanchez-Andrada, P.; Vidal, A. Org. Lett. 2006, 8, 5645-5648. doi:10.1021/ol062373w

20. Alajarin, M.; Bonillo, B.; Ortin, M.-M.; Sanchez-Andrada, P.; Vidal, A. Eur. J. Org. Chem. 2011, 1896-1913. doi:10.1002/ejoc.201001372

21. Alajarin, M.; Bonillo, B.; Sanchez-Andrada, P.; Vidal, A.; Bautista, D. Org. Lett. 2009, 11, 1365-1368. doi:10.1021/ol9001416

22. Alajarin, M.; Bonillo, B.; Sanchez-Andrada, P.; Vidal, A. J. Org. Chem. 2010, 75, 3737-3750. doi:10.1021/jo100502p

23. Alajarin, M.; Bonillo, B.; Marin-Luna, M.; Sanchez-Andrada, P.; Vidal, A.; Orenes, R.-A. Tetrahedron 2012, 68, 4672-4681. doi:10.1016/j.tet.2012.04.021

24. Alajarin, M.; Bonillo, B.; Orenes, R.-A.; Ortin, M.-M.; Vidal, A. Org. Biomol. Chem. 2012, 10, 9523-9537. doi:10.1039/c2ob27010b

25. Vidal, A.; Marin-Luna, M.; Alajarin, M. Eur. J. Org. Chem. 2014, 878-886. doi:10.1002/ejoc.201301501

26. Alajarin, M.; Marin-Luna, M.; Ortin, M.-M.; Sanchez-Andrada, P.; Vidal, A. Tetrahedron 2011, 67, 5590-5595. doi:10.1016/j.tet.2011.05.119

27. Alajarin, M.; Marin-Luna, M.; Vidal, A. Adv. Synth. Catal. 2011, 353, 553-557. doi:10.1002/adsc.201000812 
28. Strohfeldt, K.; Tacke, M. Chem. Soc. Rev. 2008, 37, 1174-1187. doi:10.1039/b707310k

29. Suzuka, T.; Ogasawara, M.; Hayashi, T. J. Org. Chem. 2002, 67, 3355-3359. doi:10.1021/jo0111199

30. Ho, T.-I.; Ho, J.-H.; Wu, J.-Y. J. Am. Chem. Soc. 2000, 122, 8575-8576. doi:10.1021/ja0011562

31. Ho, T.-I.; Wu, J.-Y.; Wang, S.-L. Angew. Chem., Int. Ed. 1999, 38, 2558-2560. doi:10.1002/(SICI)1521-3773(19990903)38:17<2558::AID-ANIE2558>3 0.CO;2-E

32. Heller, H. G.; Jenkins, G. A. J. Chem. Soc., Perkin Trans. 1 1984, 2871-2875. doi:10.1039/p19840002871

33. Stone, K. J.; Little, R. D. J. Org. Chem. 1984, 49, 1849-1853. doi:10.1021/jo00185a001

34. Roth, W. R. Tetrahedron Lett. 1964, 1009-1013. doi:10.1016/S0040-4039(00)90422-2

35. Koelsch, C. F.; Johnson, P. R. J. Am. Chem. Soc. 1943, 65, 567-573. doi:10.1021/ja01244a021

36. Miller, L. L.; Greisinger, R.; Boyer, R. F. J. Am. Chem. Soc. 1969, 91 , 1578-1580. doi:10.1021/ja01034a076

37. Rakita, P. E.; Taylor, G. A. Inorg. Chem. 1972, 11, $2136-2141$. doi:10.1021/ic50115a029

38. Almy, J.; Cram, D. J. J. Am. Chem. Soc. 1970, 92, 4316-4320. doi:10.1021/ja00717a031

39. Rakita, P. E.; Taylor, G. A. J. Organomet. Chem. 1973, 61, 71-81. doi:10.1016/S0022-328X(00)86536-5

40. Jones, D. W.; Marmon, R. J. J. Chem. Soc., Perkin Trans. 1 1993, 681-690. doi:10.1039/p19930000681

41. Rupert, K. C.; Liu, C. C.; Nguyen, T. T.; Whitener, M. A.; Sowa, J. R. Organometallics 2002, 21, 144-149. doi:10.1021/om010731n

42. Ohlsson, L.; Wallmark, I.; Bergson, G. Acta Chem. Scand. 1966, 20, 750-753. doi:10.3891/acta.chem.scand.20-0750

43. Weidler, A. M.; Bergson, G. Acta Chem. Scand. 1964, 18, 1487-1497. doi:10.3891/acta.chem.scand.18-1487

44. Friedrich, E. C.; Taggart, D. B. J. Org. Chem. 1975, 40, 720-723. doi:10.1021/jo00894a012

45. Bergson, G.; Weidler, A. M. Acta Chem. Scand. 1963, 17, 862-864. doi:10.3891/acta.chem.scand.17-0862

46. Bergson, G.; Weidler, A. M. Acta Chem. Scand. 1963, 17, 1798-1799. doi:10.3891/acta.chem.scand.17-1798

47. Bergson, G. Acta Chem. Scand. 1963, 17, 2691-2700. doi:10.3891/acta.chem.scand.17-2691

48. The relative electronic and Gibbs energies and the energy barriers computed in gas phase and in DMSO are gathered in Table S2 of Supporting Information File 2.

49. We have also found one additional transition structure for each one of the electrocyclizations $10 a \rightarrow 12 a$ and $11 a \rightarrow 13 a$, those corresponding to conrotatory processes. As presumed, they are higher in energy than its disrotatory counterparts. With the aim of simplifying, they have been omitted from this discussion.

50. The assistance of one or more water molecules in a concerted process has been also envisaged but not computationally scrutinized.

\section{License and Terms}

This is an Open Access article under the terms of the Creative Commons Attribution License

(http://creativecommons.org/licenses/by/2.0), which permits unrestricted use, distribution, and reproduction in any medium, provided the original work is properly cited.

The license is subject to the Beilstein Journal of Organic Chemistry terms and conditions:

(http://www.beilstein-journals.org/bjoc)

The definitive version of this article is the electronic one which can be found at: doi:10.3762/bjoc. 12.28 